\title{
Monotone and Concave Positive Solutions to Three-Point Boundary Value Problems of Higher-Order Fractional Differential Equations
}

\author{
Wenyong Zhong and Lanfang Wang \\ College of Mathematics and Statistics, Jishou University, 120 Renmin South Road, Jishou, Hunan 416000, China \\ Correspondence should be addressed to Wenyong Zhong; wyzhong@jsu.edu.cn
}

Received 26 June 2014; Accepted 21 August 2014

Academic Editor: Wei-Shih Du

Copyright (C) 2015 W. Zhong and L. Wang. This is an open access article distributed under the Creative Commons Attribution License, which permits unrestricted use, distribution, and reproduction in any medium, provided the original work is properly cited.

We study the three-point boundary value problem of higher-order fractional differential equations of the form ${ }^{c} D_{0+}^{\rho} u(t)+$ $f(t, u(t))=0,0<t<1,2 \leqslant n-1<\rho<n, u^{\prime}(0)=u^{\prime \prime}(0)=\cdots=u^{(n-1)}(0)=0, u(1)+p u^{\prime}(1)=q u^{\prime}(\xi)$, where ${ }^{c} D_{0+}^{\rho}$ is the Caputo fractional derivative of order $\rho$, and the function $f:[0,1] \times[0, \infty) \mapsto[0,+\infty)$ is continuously differentiable. Here, $0 \leqslant q \leqslant p, 0<\xi<1,2 \leqslant n-1<\rho<n$. By virtue of some fixed point theorems, some sufficient criteria for the existence and multiplicity results of positive solutions are established and the obtained results also guarantee that the positive solutions discussed are monotone and concave.

\section{Introduction}

Applications of fractional differential equations can be found in various areas, including engineering, physics, and chemistry [1-4]. In recent years, the interest in the study of fractional differential equations has been growing rapidly.

As one of the focal topics in the research of fractional differential equations, the study of the boundary value problems (BVPs for short) recently has attracted a great deal of attention from many researchers. A series of works have been presented to discuss the existence of (positive) solutions in the BVPs for fractional differential equations [5-15].

However, there are few results in the literature to discuss the positive, monotone, and concave solutions to the BVPs of fractional differential equations; it is difficult to establish the relation between the monotonicity and concavity of a function and its fractional derivatives. It is worth pointing out that Wang et al. [7] obtained the existence and multiplicity results of the positive, monotone, and concave solutions to the following problem:

$$
\begin{gathered}
{ }^{c} D_{0+}^{\rho} u(t)+f(t, u(t))=0, \quad 0<t<1, n-1<\rho<n, \\
u(0)=u^{\prime \prime}(0)=\cdots=u^{(n-1)}(0)=0, \\
u^{\prime}(1)=0,
\end{gathered}
$$

where ${ }^{c} D_{0+}^{\rho}$ is the Caputo fractional derivative of order $\rho$. The multiplicity results of solutions are obtained by using the Legget-Williams fixed point theorem. However, the question of how to establish the connection between the monotonicity and concavity of a function and its fractional derivatives is far from being solved; and the concavity of a function is also not used sufficiently.

Motivated by the aforementioned results, we then turn to investigating the existence of monotone and concave positive solutions for the following boundary value problem (BVP for short):

$$
\begin{gathered}
{ }^{c} D_{0+}^{\rho} u(t)+f(t, u(t))=0, \quad 0<t<1, n-1<\rho<n, \\
u^{\prime}(0)=u^{\prime \prime}(0)=\cdots=u^{(n-1)}(0)=0, \\
u(1)+p u^{\prime}(1)=q u^{\prime}(\xi),
\end{gathered}
$$

where ${ }^{c} D_{0+}^{\rho}$ is the Caputo fractional derivative of order $\rho$. The case $\rho=2$ was discussed in [16] by virtue of the AveryHenderson and Legget-Williams fixed point theorems. While in the setting of the fractional-order derivatives, as far as we 
know, the existence of positive solutions for BVP (2) has not been discussed in the literature.

We now make the following assumptions to be used later:

(A1) the function $f:[0,1] \times[0, \infty) \mapsto[0,+\infty)$ is continuously differentiable;

(A2) $0 \leqslant q \leqslant p, 0<\xi<1,2 \leqslant n-1<\rho<n$.

The rest of paper is organized as follows. Section 2 preliminarily provides some definitions and lemmas which are crucial to the following discussion, and the connection between the monotonicity and concavity of a function and its Caputo derivatives is established in this section. Section 3 gives some sufficient conditions for the existence of at least two positive solutions of BVP (2) by means of the Avery-Henderson fixed point theorem. Section 4 gives some sufficient conditions for the existence of at least three positive solutions by virtue of the five-functional fixed point theorem. In addition, the sufficient conditions also guarantee that the positive solutions obtained are monotone and concave. Finally, Section 5 provides an example to illustrate a possible application of the obtained results.

\section{Preliminaries}

In this section, we preliminarily provide some definitions and lemmas to be used in the following discussion.

Definition 1 (see [3]). The fractional integral of order $\rho>0$ of a function $y:(0, \infty) \mapsto \mathbb{R}$ is given by

$$
I_{0+}^{\rho} y(t)=\frac{1}{\Gamma(\rho)} \int_{0}^{t}(t-s)^{\rho-1} y(s) d s
$$

provided the right side is pointwise defined on $(0, \infty)$.

Definition 2 (see [3]). The Riemann-Liouville fractional derivative of order $\rho>0$ of a continuous function $y$ : $(0, \infty) \mapsto \mathbb{R}$ is given by

$$
D_{0+}^{\rho} y(t)=\frac{1}{\Gamma(n-\rho)} \frac{d^{n}}{d t^{n}} \int_{0}^{t}(t-s)^{n-\rho-1} y(s) d s
$$

where $n=[\rho]+1$, provided the right side is pointwise defined on $(0, \infty)$.

Remark 3. Consider $D_{0+}^{m} y(t)=D^{m} y(t)$, where $D^{m} y(t) \triangleq$ $d^{m} y(t) / d t^{m}, m \in N_{0} \triangleq\{0,1,2, \ldots\}$.
Definition 4 (see [4]). For a function $y$ given on the interval $[0, \infty)$, the Caputo fractional derivative of order $\rho>0$ of $y$ is defined by

$$
{ }^{c} D_{0+}^{\rho} y(t)=\frac{1}{\Gamma(n-\rho)} \int_{0}^{t}(t-s)^{n-\rho-1} y^{(n)}(s) d s,
$$

where $n=[\rho]+1,[\rho]$ denotes the integer part of $\rho$.

Lemma 5 (see [3]). Let $\rho$ and $\mu$ be positive numbers. If $y \in C[0,1]$, then $I_{0+}^{\rho} y(t) \in C[0,1]$, and the equations $D_{0+}^{\rho} I_{0+}^{\rho} y(t)=y(t)$ and $I_{0+}^{\rho} I_{0+}^{\mu} y(t)=I_{0+}^{\rho+\mu} y(t)$ are satisfied for each $t$ in $[0,1]$.

Lemma 6 (see [3]). Let $\rho>0$. If $y \in C^{n}[0,1]$ or $y \in$ $A C^{n}[0,1]$, then

$$
I_{0+}^{\rho}{ }^{c} D_{0+}^{\rho} y(t)=y(t)+c_{0}+c_{1} t+c_{2} t^{2}+\cdots+c_{n-1} t^{n-1},
$$

for some $c_{i}$ in $\mathbb{R}, i=0,1, \ldots, n-1, n=[\rho]+1$.

The following two lemmas are fundamental in finding an integral representation of solutions of BVP (2).

Lemma 7. Let $g:[0,1] \times \mathbb{R} \mapsto \mathbb{R}$ be a continuously differentiable function. If a function $y$ in $C[0,1]$ is a solution of the equation $u(t)=I_{0+}^{\rho} g(t, u(t))$, then $y \in C^{(n-1)}[0,1]$ and $y^{(n)} \in C(0,1] \cap L(0,1]$, and the relation ${ }^{c} D_{0+}^{\rho} y(t)=g(t, y(t))$ holds for each $t$ in $[0,1]$.

Proof. Let $y \in C[0,1]$ be a solution of the equation $u(t)=$ $I_{0+}^{\rho} g(t, u(t))$.

Since $g$ is continuous on $[0,1] \times \mathbb{R}$, Lemma 5 implies that

$$
I_{0+}^{\rho-1} g(t, y(t)) \in C[0,1]
$$

and that

$$
I_{0+}^{1} I_{0+}^{\rho-1} g(t, y(t))=I_{0+}^{\rho} g(t, y(t))
$$

The above equation, together with Remark 3 and Lemma 5, yields

$$
D_{0+}^{1} I_{0+}^{1} I_{0+}^{\rho-1} g(t, y(t))=I_{0+}^{\rho-1} g(t, y(t)) .
$$

Hence $y(t)$ is continuously differentiable on $[0,1]$ and $y^{\prime}(t)=$ $I_{0+}^{\rho-1} g(t, y(t))$.

Generally, noticing $n-1<\rho<n$, we have

$$
y^{(k)}(t)=I_{0+}^{\rho-k} g(t, y(t))
$$

which implies $y^{(k)} \in C[0,1]$ for $k=1,2, \ldots, n-1$. 
Furthermore, using the assumptions imposed on the function $g(t, u)$ and integrating by parts, we obtain

$$
\begin{aligned}
y^{(n-1)}(t) & \\
= & \frac{1}{\Gamma(\rho-n+1)} \int_{0}^{t}(t-s)^{\rho-n} g(s, y(s)) d s \\
= & \frac{g(0, y(0))}{\Gamma(\rho-n+2)} t^{\rho-n+1} \\
& +\frac{1}{\Gamma(\rho-n+2)} \int_{0}^{t}(t-s)^{\rho-n+1}\left(\frac{\partial g}{\partial s}+y^{\prime}(s) \frac{\partial g}{\partial y}\right) d s \\
= & \frac{g(0, y(0))}{\Gamma(\rho-n+2)} t^{\rho-n+1}+I_{0+}^{\rho-n+2}\left(\frac{\partial g}{\partial t}+y^{\prime}(t) \frac{\partial g}{\partial y}\right) .
\end{aligned}
$$

This yields that, for every $t$ in $(0,1]$,

$$
y^{(n)}(t)=\frac{g(0, y(0))}{\Gamma(\rho-n+1)} t^{\rho-n}+I_{0+}^{\rho-n+1}\left(\frac{\partial g}{\partial t}+y^{\prime}(t) \frac{\partial g}{\partial y}\right) .
$$

Since the second term of the right-hand side of the above equality is continuous on the interval $[0,1], y^{(n)} \in C(0,1] \cap$ $L(0,1]$. Consequently, direct computations produce

$$
\begin{aligned}
{ }^{c} D_{0+}^{\rho} y(t)= & I_{0+}^{n-\rho} y^{(n)}(t) \\
= & \frac{g(0, y(0))}{\Gamma(\rho-n+1)} I_{0+}^{n-\rho} t^{\rho-n} \\
& +I_{0+}^{n-\rho} I_{0+}^{\rho-n+1}\left(\frac{\partial g}{\partial t}+y^{\prime}(t) \frac{\partial g}{\partial y}\right) \\
= & g(0, y(0))+I_{0+}^{1} D g(t, y(t)) \\
= & g(t, y(t)) .
\end{aligned}
$$

The proof is completed.

By Lemma 6, we next present an integral representation of the solution of the linearized problem corresponding to BVP (2).

Lemma 8. Let $h \in C^{1}[0,1]$; if $(A 1)-(A 2)$ hold, then $B V P$

$$
\begin{gathered}
{ }^{c} D_{0+}^{\rho} u(t)+h(t)=0, \quad 0<t<1,2 \leqslant n-1<\rho<n, \\
u^{\prime}(0)=u^{\prime \prime}(0)=\cdots=u^{(n-1)}(0)=0, \\
u(1)+p u^{\prime}(1)=q u^{\prime}(\xi)
\end{gathered}
$$

has a unique solution

$$
u(t)=\int_{0}^{1} G(t, s) h(s) d s
$$

where

$$
\begin{gathered}
G(t, s)=G_{1}(t, s)+G_{2}(t, s) . \\
G_{1}(t, s)= \begin{cases}\frac{(1-s)^{\rho-1}-(t-s)^{\rho-1}}{\Gamma(\rho)}, & s \leqslant t, \\
\frac{(1-s)^{\rho-1}}{\Gamma(\rho)}, & s \geqslant t,\end{cases} \\
G_{2}(t, s)=\frac{1}{\Gamma(\rho-1)}\left[p(1-s)^{\rho-2}-q(\xi-s)^{\rho-2} \chi_{E_{\xi}}(s)\right] .
\end{gathered}
$$

Here $E_{\xi}=\{s: s \leqslant \xi\}$, and $\chi_{E_{\xi}}$ denotes the characteristic function of the set $E_{\xi}$.

Proof. Lemma 6 implies

$$
u(t)=-I_{0+}^{\rho} h(t)+c_{0}+c_{1} t+c_{2} t^{2}+\cdots+c_{n-1} t^{n-1} .
$$

Differentiating (19) with respect to $t$ up to the order $n-1$ and using the boundary conditions that $u^{\prime}(0)=u^{\prime \prime}(0)=\cdots=$ $u^{(n-1)}(0)=0$, we obtain

$$
u(t)=-I_{0+}^{\rho} h(t)+c_{0}
$$

From the above equation and the condition that $u(1)+$ $p u^{\prime}(1)=q u^{\prime}(\xi)$, it follows that

$$
c_{0}=I_{0+}^{\rho} h(1)+p I_{0+}^{\rho-1} h(1)-q I_{0+}^{\rho-1} h(\xi) .
$$

Substituting $c_{0}$ into (20), we have

$$
\begin{aligned}
u(t)= & -I^{\rho} h(t)+I_{0+}^{\rho} h(1)+p I_{0+}^{\rho-1} h(1)-q I_{0+}^{\rho-1} h(\xi) \\
= & \int_{0}^{t} \frac{(1-s)^{\rho-1}-(t-s)^{\rho-1}}{\Gamma(\rho)} h(s) d s \\
& +\int_{t}^{1} \frac{(1-s)^{\rho-1}}{\Gamma(\rho)} h(s) d s \\
& +p \int_{0}^{1} \frac{(1-s)^{\rho-2}}{\Gamma(\rho-1)} h(s) d s-q \int_{0}^{\xi} \frac{(\xi-s)^{\rho-2}}{\Gamma(\rho-1)} h(s) d s \\
= & \int_{0}^{1} G(t, s) h(s) d s
\end{aligned}
$$

where $G(t, s)$ is defined by (16). The proof is completed.

We now give some properties of the functions $G_{i}(t, s)$.

Lemma 9. If condition (A2) holds, then

$$
G_{i}(s, s) \geqslant G_{i}(t, s) \geqslant \eta(t) G_{i}(s, s) \geqslant 0,
$$

for all $s, t$ in $[0,1]$ and $i=1,2$, where $\eta(t)=1-t^{\rho-1}$. 
Proof. It follows from the definition of $G_{1}(t, s)$ that, for $t \geqslant s$,

$$
\begin{aligned}
G_{1}(s, s) & \geqslant G_{1}(t, s)=\frac{(1-s)^{\rho-1}-(t-s)^{\rho-1}}{\Gamma(\rho)} \\
& \geqslant \frac{(1-s)^{\rho-1}-(t-t s)^{\rho-1}}{\Gamma(\rho)} \\
& =\eta(t) G_{1}(s, s) \geqslant 0 .
\end{aligned}
$$

On the other hand, for $t \leqslant s$, the assertion for $G_{1}(t, s)$ is obvious.

As for the assertion for $G_{2}(t, s)$, it is sufficient to verify that $G_{2}(s, s) \geqslant 0$ for each $s$ in $[0,1]$. In fact, the definition of $G_{2}(t, s)$ and condition (A2) directly imply

$$
\begin{aligned}
G_{2}(s, s) & =\frac{1}{\Gamma(\rho-1)}\left[p(1-s)^{\rho-2}-q(\xi-s)^{\rho-2} \chi_{E_{\xi}}(s)\right] \\
& \geqslant \frac{1}{\Gamma(\rho-1)}\left[p(1-s)^{\rho-2}-q(1-s)^{\rho-2}\right] \\
& =\frac{p-q}{\Gamma(\rho-1)}(1-s)^{\rho-2} \geqslant 0 .
\end{aligned}
$$

The proof is completed.

The following results establish the connection between the monotonicity and concavity of a function and its Caputo fractional derivatives under some conditions.

Lemma 10. Let $u$ be a function defined on [0,1]. Assume $u^{(k)}(0)=0$ for $k=1,2, \ldots, n-1$. Suppose that ${ }^{c} D_{0+}^{\rho} u(t)$ is continuously differentiable on $[0,1]$; if ${ }^{c} D_{0+}^{\rho} u(t) \leqslant 0$ on $[0,1]$, then $u^{(k)}(t) \leqslant 0$ on $[0,1]$ for $k=1,2, \ldots, n-1$.

Proof. Set $h(t)={ }^{c} D_{0+}^{\rho} u(t)$. Then, as in the proof of Lemma 8, the assumptions made on ${ }^{c} D_{0+}^{\rho} u(t)$ and $u^{k}(0)$ yield

$$
u(t)=I_{0+}^{\rho} h(t)+c_{0} .
$$

This implies

$$
u^{(k)}(t)=I_{0+}^{\rho-k} h(t),
$$

for $k=1,2, \ldots n-1$. Thus the desired results follow from the nonpositivty of $h(t)$. The proof is completed.

Lemmas 8-10 yield the following important properties of the solution of BVP (14), which is easy to check.

Lemma 11. Let $h \in C^{1}([0,1],[0,+\infty))$. If condition (A2) holds, then the solution of BVP (14) is nonnegative, monotone, and concave on $[0,1]$.

Lemma 12 (see [17]). If a function $u$ is nonnegative and concave on $[0,1]$ and $u^{\prime}(0)=0$, then

(i) $u(t) \geqslant(1-t)\|u\|$ for each $t$ in $[0,1]$, where $\|u\|=$ $\sup \{|u(t)|: t \in[0,1]\}$

(ii) $(1-s) u(t) \geqslant(1-t) u(s)$ for all $t, s$ in $[0,1]$ with $t \geqslant s$.
Now, denote by $\mathscr{E}=C[0,1]$ the classical Banach space with the norm $\|u\|=\sup _{t \in[0,1]}|u(t)|$, where $u \in \mathscr{E}$. Furthermore, define a cone, denoted by $\mathscr{P}$, through

$$
\begin{gathered}
\mathscr{P}=\left\{u \in \mathscr{E} \mid u(t) \geqslant 0, u^{\prime}(t) \leqslant 0, u^{\prime \prime}(t) \leqslant 0\right. \\
\text { for each } t \text { in }[0,1]\} .
\end{gathered}
$$

Also, for a given positive real number $r$, define a function set $\mathscr{P}_{r}$ by

$$
\mathscr{P}_{r}=\{u \in \mathscr{P} \mid\|u\|<r\}
$$

Naturally, we denote that $\overline{\mathscr{P}}_{r}=\{u \in \mathscr{P} \mid\|u\| \leqslant r\}$ and that $\partial \mathscr{P}_{r}=\{u \in \mathscr{P} \mid\|u\|=r\}$.

Next, define the operator $\mathscr{A}: \mathscr{P} \rightarrow \mathscr{E}$ by

$$
[\mathscr{A} u](t)=\int_{0}^{1} G(t, s) f(s, u(s)) d s,
$$

for any $u \in \mathscr{P}$. We now show some important properties on this map.

Lemma 13. Assume that hypotheses (A1)-(A2) are all fulfilled. Then $\mathscr{A}(\mathscr{P}) \subset \mathscr{P}$ and $\mathscr{A}: \overline{\mathscr{P}}_{r} \rightarrow \mathscr{P}$ is completely continuous.

Proof. It is easy to check that $\mathscr{A}(\mathscr{P}) \subset \mathscr{P}$. Moreover, analysis similar to that in [6] shows that $\mathscr{A}: \overline{\mathscr{P}}_{r} \rightarrow \mathscr{P}$ is completely continuous. The proof is completed.

Lemma 14. If (A1)-(A2) hold, then a function $u$ in $C[0,1]$ is a solution of BVP (2) if and only if it is a fixed point of $\mathscr{A}$ in $\mathscr{P}$.

Proof. If $u$ is a solution of BVP (2), then Lemma 11 implies $u \in \mathscr{P}$. Furthermore, replacing $h(t)$ in Lemma 8 by $f(t, u(t))$, we get $\mathscr{A} u=u$. Hence $u$ is a fixed point of $\mathscr{A}$ in $\mathscr{P}$.

On the other hand, if $u \in \mathscr{P}$ and $\mathscr{A} u=u$, then

$$
\begin{aligned}
u(t)= & \int_{0}^{1} G(t, s) f(s, u(s)) d s \\
= & -I_{0+}^{\rho} f(t, u(t))+I_{0+}^{\rho} f(1, u(1)) \\
& +p I_{0+}^{\rho-1} f(1, u(1))-q I_{0+}^{\rho-1} f(\xi, u(\xi)) .
\end{aligned}
$$

The above equation and Lemma 7 imply

$$
D_{0+}^{\rho} u(t)+f(t, u(t))=0 .
$$

Moreover, it is easy to check that all the boundary conditions in BVP (2) are satisfied. Therefore $u$ is a positive solution of BVP (2). We consequently complete the proof.

\section{Two Positive Solutions in Boundary Value Problems}

In this section, we aim to adopt the well-known AveryHenderson fixed point theorem to prove the existence of at least two positive solutions in BVP (2). For the sake of selfcontainment, we first state the Avery-Henderson fixed point theorem as follows. 
Theorem 15 (see [18]). Let $\mathscr{P}$ be a cone in a real Banach space $\mathscr{E}$. For each $d>0$, set $\mathscr{P}(\psi, d)=\{x \in \mathscr{P} \mid \psi(x)<d\}$. Let $\alpha$ and $\gamma$ be increasing, nonnegative continuous functional on $\mathscr{P}$, and let $\theta$ be a nonnegative continuous functional on $\mathscr{P}$ with $\theta(0)=0$ such that, for some $c>0$ and $H>0$,

$$
\gamma(x) \leqslant \theta(x) \leqslant \alpha(x), \quad\|x\| \leqslant H \gamma(x)
$$

for all $x \in \overline{\mathscr{P}(\gamma, c)}$. Suppose that there exist a completely continuous operator $\mathscr{A}: \overline{\mathscr{P}(\gamma, c)} \rightarrow \mathscr{P}$ and three positive numbers $0<a<b<c$ such that

$$
\theta(\lambda x) \leqslant \lambda \theta(x), \quad 0 \leqslant \lambda \leqslant 1, \quad x \in \partial \mathscr{P}(\theta, b),
$$

and (i) $\gamma(\mathscr{A} x)>c$ for all $x \in \partial \mathscr{P}(\gamma, c)$; (ii) $\theta(\mathscr{A} x)<b$ for all $x \in \partial \mathscr{P}(\theta, b)$; (iii) $\mathscr{P}(\alpha, a) \neq \emptyset$ and $\alpha(\mathscr{A} x)>$ a for all $x \in$ $\partial \mathscr{P}(\alpha, a)$. Then, the operator $\mathscr{A}$ has at least two fixed points, denoted by $x_{1}$ and $x_{2}$, belonging to $\overline{\mathscr{P}(\gamma, c)}$ and satisfying $a<$ $\alpha\left(x_{1}\right)$ with $\theta\left(x_{1}\right)<b$ and $b<\theta\left(x_{2}\right)$ with $\gamma\left(x_{2}\right)<c$.

Now, select $t_{\star}$ and $t^{\star}$ such that $0<t_{\star}<1 / 2<t^{\star}<1$. Let

$$
\begin{gathered}
M=\frac{\left(1-t^{\star}\right)\left(1-\left(1-t^{\star}\right)^{\rho}\right)}{\Gamma(\rho+1)}, \\
N=\frac{p \rho+1}{\Gamma(\rho+1)}, \\
L=\frac{\left(1-t_{\star}\right)^{\rho+1}}{\Gamma(\rho+1)}, \\
L_{0}=\frac{1+p \rho-t_{\star}^{\rho}}{\Gamma(\rho+1)} .
\end{gathered}
$$

We are now in a position to obtain the following result.

Theorem 16. Assume that hypotheses (A1)-(A2) all hold and that there exist positive real numbers $a, b$, and $c$ such that

$$
0<a<b<c, \quad a<\frac{L}{N} b<\frac{L\left(1-t^{\star}\right)}{N} c .
$$

Furthermore, assume that $f$ satisfies the following conditions:

$$
\begin{aligned}
& \text { (C1) } f(t, u)>c / M \text { for }(t, u) \text { in }\left[0, t^{\star}\right] \times\left[c,\left(1 /\left(1-t^{\star}\right)\right) c\right] \text {; } \\
& \text { (C2) } f(t, u)<b / N \text { for }(t, u) \text { in }[0,1] \times\left[0,\left(1 /\left(1-t^{\star}\right)\right) b\right] \text {; } \\
& \text { (C3) } f(t, u)>a / L \text { for }(t, u) \text { in }\left[t_{\star}, 1\right] \times[0, a] \text {. }
\end{aligned}
$$

Then BVP (2) has at least two positive solutions $u_{1}$ and $u_{2}$ such that

$$
\begin{aligned}
& a<\max _{t \in\left[t_{\star}, 1\right]} u_{1}(t) \quad \text { with } \max _{t \in\left[t^{\star}, 1\right]} u_{1}(t)<b, \\
& b<\max _{t \in\left[t^{\star}, 1\right]} u_{1}(t) \quad \text { with } \min _{t \in\left[t_{\star}, t^{\star}\right]} u_{2}(t)<c .
\end{aligned}
$$

Proof. Let the cone $\mathscr{P}$ and the operator $\mathscr{A}$ be defined by (28) and (30), respectively. Furthermore, define the increasing, nonnegative, and continuous functionals $\gamma, \theta$, and $\alpha$ on $\mathscr{P}$, respectively, by

$$
\begin{aligned}
& \gamma(u)=\min _{t \in\left[t_{\star}, t^{\star}\right]} u(t)=u\left(t^{\star}\right), \\
& \theta(u)=\max _{t \in\left[t^{\star}, 1\right]} u(t)=u\left(t^{\star}\right), \\
& \alpha(u)=\max _{t \in\left[t_{\star}, 1\right]} u(t)=u\left(t_{\star}\right) .
\end{aligned}
$$

Evidently, $\gamma(u)=\theta(u) \leqslant \alpha(u)$ for each $u$ in $\mathscr{P}$.

Moreover, for each $u$ in $\mathscr{P}$, Lemma 12 implies that $u\left(t^{\star}\right) \geqslant$ $\left(1-t^{\star}\right)\|u\|$. Observing $\gamma(u)=u\left(t^{\star}\right)$, we have

$$
\|u\| \leqslant \frac{1}{1-t^{\star}} \gamma(u)
$$

for each $u$ in $\mathscr{P}$. Also, notice that $\theta(\lambda u)=\lambda \theta(u)$ for each $\lambda$ in $[0,1]$ and $u$ in $\partial \mathscr{P}(\theta, b)$. In addition, Lemma 13 guarantees that the operator $\mathscr{A}: \overline{\mathscr{P}(\gamma, c)} \rightarrow \mathscr{P}$ is completely continuous.

Next, we are to verify that all the conditions of Theorem 15 are satisfied with respect to the operator $\mathscr{A}$.

Let $u \in \partial \mathscr{P}(\gamma, c)$. Then $\gamma(u)=\min _{t \in\left[t_{\star}, t^{\star}\right]} u(t)=u\left(t^{\star}\right)=$ $c$. This implies that $u(t) \geqslant c$ for each $t$ in $\left[0, t^{\star}\right]$, which, combined with (39), yields that

$$
c \leqslant u(t) \leqslant \frac{1}{1-t^{\star}} c,
$$

for each $t$ in $\left[0, t^{\star}\right]$. This inequality and assumption (C1) imply

$$
f(t, u(t))>\frac{c}{M}
$$

for each $t$ in $\left[0, t^{\star}\right]$. Now, from the definition of the operator $\mathscr{A}$ and Lemmas 8 and 12 , we obtain that

$$
\begin{aligned}
\gamma(\mathscr{A} u) & =[\mathscr{A} u]\left(t^{\star}\right) \geqslant\left(1-t^{\star}\right)\|\mathscr{A} u\|=\left(1-t^{\star}\right)[\mathscr{A} u](0) \\
& =\left(1-t^{\star}\right) \int_{0}^{1} G(0, s) f(s, u(s)) d s \\
& >\left(1-t^{\star}\right) \cdot \frac{c}{M} \cdot \int_{0}^{t^{\star}} G_{1}(0, s) d s \\
& >\left(1-t^{\star}\right) \cdot \frac{c}{M} \cdot \frac{1-\left(1-t^{\star}\right)^{\rho}}{\Gamma(\rho+1)}=c .
\end{aligned}
$$

Thus condition (i) in Theorem 15 is satisfied.

We now claim that condition (ii) in Theorem 15 is satisfied. To this end, let $u \in \partial \mathscr{P}(\theta, b)$. Then, $\gamma(u)=\theta(u)=$ $\max _{t \in\left[t^{\star}, 1\right]} u(t)=u\left(t^{\star}\right)=b$, from which we have $0 \leqslant u(t) \leqslant b$ for each $t$ in $\left[t^{\star}, 1\right]$. Analogously, it follows from inequality (39) that, for each $u$ in $\mathscr{P}$,

$$
\|u\| \leqslant \frac{1}{1-t^{\star}} \gamma(u)=\frac{1}{1-t^{\star}} b,
$$


which implies $0 \leqslant u(t) \leqslant\left(1 /\left(1-t^{\star}\right)\right) b$ for each $t$ in $[0,1]$. This, combined with assumption (C2), yields $f(t, u(t))<$ $b / N$ for each $t$ in $[0,1]$. Thus we have

$$
\begin{aligned}
\theta(\mathscr{A} u)= & \max _{t \in\left[t^{\star}, 1\right]}[\mathscr{A} u](t)=[\mathscr{A} u]\left(t^{\star}\right) \leqslant[\mathscr{A} u](0) \\
= & \int_{0}^{1} G(0, s) f(s, u(s)) d s \\
= & \int_{0}^{1} G_{1}(0, s) f(s, u(s)) d s \\
& +\int_{0}^{1} G_{2}(0, s) f(s, u(s)) d s \\
< & \frac{b}{N}\left[\int_{0}^{1} \frac{1}{\Gamma(\rho)}(1-s)^{\rho-1} d s\right. \\
& \left.+\int_{0}^{1} \frac{1}{\Gamma(\rho-1)} p(1-s)^{\rho-2} d s\right] \\
= & \frac{b}{N} \cdot \frac{1+p \rho}{\Gamma(\rho+1)}=b,
\end{aligned}
$$

which consequently implies the validity of condition (ii) in Theorem 15.

Finally, notice that the constant function $(1 / 2) a \in$ $\mathscr{P}(\alpha, a)$ so that $\mathscr{P}(\alpha, a) \neq \emptyset$. Letting $u \in \partial \mathscr{P}(\alpha, a)$, we get $\alpha(u)=\max _{t \in\left[t_{\star}, 1\right]} u(t)=u\left(t_{\star}\right)=a$. This with assumption (C3) implies that $0 \leqslant u(t) \leqslant a$ and $f(t, u)>a / L$ for each $t$ in $\left[t_{\star}, 1\right]$. Similarly, we have

$$
\begin{aligned}
\alpha(\mathscr{A} u) & =[\mathscr{A} u]\left(t_{\star}\right) \geqslant\left(1-t_{\star}\right)[\mathscr{A} u](0) \\
& =\left(1-t_{\star}\right) \int_{0}^{1} G(0, s) f(s, u(s)) d s \\
& >\left(1-t_{\star}\right) \frac{a}{L} \cdot \int_{t_{\star}}^{1} G_{1}(0, s) d s \\
& >\left(1-t_{\star}\right) \frac{a}{L} \cdot \frac{\left(1-t_{\star}\right)^{\rho}}{\Gamma(\rho+1)}=a .
\end{aligned}
$$

Thus condition (iii) in Theorem 15 is satisfied.

Consequently, an application of Theorem 15 implies that BVP (2) has at least two positive solutions, denoted by $u_{1}$ and $u_{2}$, satisfying $a<\alpha\left(u_{1}\right)$ with $\theta\left(u_{1}\right)<b$ and $b<\theta\left(u_{2}\right)$ with $\gamma\left(u_{2}\right)<c$, respectively.

\section{Three Positive Solutions in Boundary Value Problems}

In this section, we are to prove the existence of at least three positive solutions in BVP (2) by using the five-functional fixed point theorem which is attributed to Avery [19].

Let $\gamma, \beta, \theta$ be nonnegative continuous convex functionals on $\mathscr{P}$. $\alpha$ and $\psi$ are supposed to be nonnegative continuous concave functionals on $\mathscr{P}$. Thus, for nonnegative real numbers $h, a, b, c$, and $d$, define five convex sets, respectively, by

$$
\begin{gathered}
\mathscr{P}(\gamma, c)=\{x \in \mathscr{P} \mid \gamma(x)<c\}, \\
\mathscr{P}(\gamma, \alpha, a, c)=\{x \in \mathscr{P} \mid a \leqslant \alpha(x), \gamma(x) \leqslant c\}, \\
\mathcal{Q}(\gamma, \beta, d, c)=\{x \in \mathscr{P} \mid \beta(x) \leqslant d, \gamma(x) \leqslant c\}, \\
\mathscr{P}(\gamma, \theta, \alpha, a, b, c)=\{x \in \mathscr{P} \mid a \leqslant \alpha(x), \theta(x) \leqslant b, \gamma(x) \leqslant c\}, \\
\mathbb{Q}(\gamma, \beta, \psi, h, d, c)=\{x \in \mathscr{P} \mid h \leqslant \psi(x), \beta(x) \leqslant d, \gamma(x) \leqslant c\} .
\end{gathered}
$$

Theorem 17 (see [19]). Let $\mathscr{P}$ be a cone in a real Banach space $\mathscr{E}$. Suppose that $\alpha$ and $\psi$ are nonnegative continuous concave functionals on $\mathscr{P}$ and that $\gamma, \beta$, and $\theta$ are nonnegative continuous convex functionals on $\mathscr{P}$ such that, for some positive numbers $c$ and $M$,

$$
\alpha(x) \leqslant \beta(x), \quad\|x\| \leqslant M \gamma(x),
$$

for all $x \in \overline{\mathscr{P}(\gamma, c)}$. In addition, suppose that $\mathscr{A}: \overline{\mathscr{P}(\gamma, c)} \mapsto$ $\overline{\mathscr{P}}(\gamma, c)$ is a completely continuous operator and that there exist nonnegative real numbers $h, d, a, b$ with $0<d<a$ such that

(i) $\{x \in \mathscr{P}(\gamma, \theta, \alpha, a, b, c) \mid \alpha(x)>a\} \neq \emptyset$ and $\alpha(\mathscr{A} x)>a$ for $x \in \mathscr{P}(\gamma, \theta, \alpha, a, b, c)$;

(ii) $\{x \in \mathscr{Q}(\gamma, \beta, \psi, h, d, c) \mid \beta(x)<d\} \neq \emptyset$ and $\beta(\mathscr{A} x)<d$ for $x \in \mathbb{Q}(\gamma, \beta, \psi, h, d, c)$;

(iii) $\alpha(\mathscr{A} x)>$ a for $x \in \mathscr{P}(\gamma, \alpha, a, c)$ with $\theta(\mathscr{A} x)>b$;

(iv) $\beta(\mathscr{A} x)<d$ for $x \in \mathbb{Q}(\gamma, \beta, d, c)$ with $\psi(\mathscr{A} x)<h$.

Then the operator $\mathscr{A}$ admits at least three fixed points $x_{1}, x_{2}$, and $x_{3} \in \overline{\mathscr{P}}(\gamma, c)$ satisfying $\beta\left(x_{1}\right)<d, a<\alpha\left(x_{2}\right)$, and $d<$ $\beta\left(x_{3}\right)$ with $\alpha\left(x_{3}\right)<a$, respectively.

With this theorem, we are now in a position to establish the following result on the existence of at least three positive solutions in BVP (2).

Theorem 18. Suppose that hypotheses (A1)-(A2) are all fulfilled. Assume that there exist positive real numbers $a, b$, and c such that

$$
\begin{gathered}
0<a<b<c, \quad a<\left(1-t_{\star}\right) b<\left(1-t_{\star}\right)\left(1-t^{\star}\right) c, \\
N b<M c .
\end{gathered}
$$

Furthermore, assume that $f$ satisfies the following conditions:

(H1) $f(t, u)<c / N$ for $(t, u)$ in $[0,1] \times\left[0,\left(1 /\left(1-t^{\star}\right)\right) c\right]$;

(H2) $f(t, u)>b / M$ for $(t, u)$ in $\left[0, t^{\star}\right] \times\left[b,\left(1 /\left(1-t^{\star}\right)^{2}\right) b\right]$;

(H3) $f(t, u)<a / L_{0}$ for $(t, u)$ in $[0,1] \times\left[0,\left(1 /\left(1-t_{\star}\right)\right) a\right]$.

Then BVP (2) admits at least three positive solutions $u_{1}(t)$, $u_{2}(t)$, and $u_{3}(t)$, defined on $[0,1]$, satisfying, respectively,

$$
\begin{gathered}
\max _{t \in\left[t_{\star}, 1\right]} u_{1}(t)<a, \quad b<\min _{t \in\left[0, t_{\star}\right]} u_{2}(t), \\
a<\max _{t \in\left[t_{\star}, 1\right]} u_{3}(t) \quad \text { with } \min _{t \in\left[0, t_{\star}\right]} u_{3}(t)<b .
\end{gathered}
$$


Proof. Let the cone $\mathscr{P}$ and the operator $\mathscr{A}$ be defined by (28) and (30), respectively. Define, respectively, the nonnegative continuous concave functionals on the $\mathscr{P}$ as follows:

$$
\begin{gathered}
\gamma(u)=\theta(u)=\max _{t \in\left[t^{\star}, 1\right]} u(t)=u\left(t^{\star}\right), \\
\alpha(u)=\min _{t \in\left[0, t_{\star}\right]} u(t)=u\left(t_{\star}\right), \\
\beta(u)=\max _{t \in\left[t_{\star}, 1\right]} u(t)=u\left(t_{\star}\right), \\
\psi(u)=\min _{t \in\left[0, t^{\star}\right]} u(t)=u\left(t^{\star}\right) .
\end{gathered}
$$

It is obvious that $\alpha(u)=\beta(u)$ for $u$ in $\mathscr{P}$. Moreover, from Lemma 12, it follows that

$$
\|u\| \leqslant \frac{1}{1-t^{\star}} \gamma(u),
$$

for each $u$ in $\mathscr{P}$.

Next, we intend to verify that all the conditions in Theorem 17 hold with respect to the operator $\mathscr{A}$. We first claim that the operator $\mathscr{A}: \overline{\mathscr{P}}(\gamma, c) \mapsto \overline{\mathscr{P}(\gamma, c)}$ is completely continuous. By Lemma 13, we only need to show that $\mathscr{A} u \mathrm{C}$ $\overline{\mathscr{P}}(\gamma, c)$ for each $u$ in $\overline{\mathscr{P}(\gamma, c)}$. To this end, let $u \in \overline{\mathscr{P}(\gamma, c)}$. Then, $\gamma(u)=\max _{t \in\left[t^{\star}, 1\right]} u(t)=u\left(t^{\star}\right) \leqslant c$, which, combined with (51), implies that $0 \leqslant u(t) \leqslant\left(1 /\left(1-t^{\star}\right)\right) c$ for $t$ in $[0,1]$ and $u$ in $\mathscr{P}$. Thus, it follows from assumption (H1) that, for $t \in[0,1], f(t, u(t))<c / N$, from which we have the following estimations:

$$
\begin{aligned}
\|\gamma(\mathscr{A} u)\|= & {[\mathscr{A} u]\left(t^{\star}\right) \leqslant[\mathscr{A} u](0) } \\
= & \int_{0}^{1} G(0, s) f(s, u(s)) d s \\
= & \int_{0}^{1} G_{1}(0, s) f(s, u(s)) d s \\
& +\int_{0}^{1} G_{2}(0, s) f(s, u(s)) d s \\
< & \frac{c}{N}\left[\int_{0}^{1} \frac{1}{\Gamma(\rho)}(1-s)^{\rho-1} d s\right. \\
& \left.+\int_{0}^{1} \frac{1}{\Gamma(\rho-1)} p(1-s)^{\rho-2} d s\right] \\
= & \frac{1+p \rho}{N(\rho+1)}=c .
\end{aligned}
$$

Hence we obtain the desired result. Now, it remains to verify that conditions (i)-(iv) in Theorem 17 are satisfied.

Since the constant function $u(t) \equiv\left(\left(2-t^{\star}\right) / 2\left(1-t^{\star}\right)\right) b$ belongs to the set

$$
\left\{u \in \mathscr{P}\left(\gamma, \theta, \alpha, b, \frac{1}{1-t^{\star}} b, c\right) \mid \alpha(u)>b\right\},
$$

the set

$$
\left\{u \in \mathscr{P}\left(\gamma, \theta, \alpha, b, \frac{1}{1-t^{\star}} b, c\right) \mid \alpha(u)>b\right\}
$$

is not empty. Analogously, since

$u(t)$

$$
\equiv \frac{1+t^{\star}}{2} a \in\left\{u \in \mathbb{Q}\left(\gamma, \beta, \psi,\left(1-t^{\star}\right) a, a, c\right) \mid \beta(u)<a\right\},
$$

the set

$$
\left\{u \in \mathbb{Q}\left(\gamma, \beta, \psi,\left(1-t^{\star}\right) a, a, c\right) \mid \beta(u)<a\right\},
$$

is nonempty

In addition, for $u$ in $\mathscr{P}\left(\gamma, \theta, \alpha, b,\left(1 /\left(1-t^{\star}\right)\right) b, c\right)$, inequality (51) implies

$$
\begin{aligned}
b & \leqslant \min _{t \in\left[0, t^{\star}\right]} u(t)=u\left(t^{\star}\right) \leqslant u(t) \leqslant \frac{1}{1-t^{\star}} \gamma(u) \\
& =\frac{1}{\left(1-t^{\star}\right)} \theta(u) \leqslant \frac{1}{\left(1-t^{\star}\right)^{2}} b,
\end{aligned}
$$

for each $t$ in $\left[0, t^{\star}\right]$. From assumption (H2), we thus obtain

$$
f(t, u(t))>\frac{b}{M} \text { for } t \in\left[0, t^{\star}\right] .
$$

Hence, it follows from (58) and Lemma 12 that

$$
\begin{aligned}
\alpha(\mathscr{A} u) & =[\mathscr{A} u]\left(t_{\star}\right) \geqslant\left(1-t_{\star}\right)[\mathscr{A}] u(0) \\
& =\left(1-t_{\star}\right) \int_{0}^{1} G(0, s) f(s, u(s)) d s \\
& >\frac{b}{M} \cdot\left(1-t_{\star}\right) \int_{0}^{t^{\star}} G_{1}(0, s) d s=b .
\end{aligned}
$$

Therefore condition (i) in Theorem 17 is satisfied.

We next claim that condition (ii) in Theorem 17 is satisfied. To see this, letting $u \in \mathbb{Q}\left(\gamma, \beta, \psi,\left(1-t^{\star}\right) a, a, c\right)$, then we get

$$
0 \leqslant u(t) \leqslant \frac{1}{1-t_{\star}} a,
$$

for each $t$ in $[0,1]$. Thus assumption (H3) yields $f(t, u(t))<$ $a / L_{0}$. Furthermore, we have

$$
\begin{aligned}
\beta(\mathscr{A} u)= & \int_{0}^{1} G\left(t_{\star}, s\right) f(s, u(s)) d s \\
< & \frac{a}{L_{0}}\left[\int_{0}^{1} G_{1}(s, s) d s-\int_{0}^{t_{\star}} \frac{\left(t_{\star}-s\right)^{\rho-1}}{\Gamma(\rho)} d s\right. \\
& \left.+\int_{0}^{1} G_{2}\left(t_{\star}, s\right) d s\right] \\
< & \frac{a}{L_{0}}\left[\int_{0}^{1} \frac{(1-s)^{\rho-1}}{\Gamma(\rho)} d s-\int_{0}^{t_{\star}} \frac{\left(t_{\star}-s\right)^{\rho-1}}{\Gamma(\rho)} d s\right. \\
& \left.+\int_{0}^{1} \frac{p\left(t_{\star}-s\right)^{\rho-2}}{\Gamma(\rho-1)} d s\right] \\
= & \frac{a}{L_{0}} \cdot \frac{1+p \rho-t_{\star}^{\rho}}{\Gamma(\rho+1)} a .
\end{aligned}
$$


Accordingly, the validity of condition (ii) in Theorem 17 is verified.

Aside from conditions (i) and (ii), we are finally to verify the validity of conditions (iii) and (iv). For this purpose, on the one hand, consider $u \in \mathscr{P}(\gamma, \alpha, b, c)$ with $\theta(\mathscr{A} u)>$ $\left(1 /\left(1-t^{\star}\right)\right) b$. Thus we have

$$
\alpha(\mathscr{A} u)=[\mathscr{A} u]\left(t_{\star}\right) \geqslant[\mathscr{A} u]\left(t^{\star}\right)=\theta(\mathscr{A} u)>\frac{1}{1-t^{\star}} b>b .
$$

On the other hand, consider $u \in \mathbb{Q}(\gamma, \beta, a, c)$ with $\psi(\mathscr{A} u)<$ $\left(1-t^{\star}\right) a$. In such a case, we obtain

$$
\begin{aligned}
\beta(\mathscr{A} u) & =[\mathscr{A} u]\left(t_{\star}\right) \leqslant \frac{1-t_{\star}}{1-t^{\star}}[\mathscr{A} u]\left(t^{\star}\right) \\
& =\frac{1-t_{\star}}{1-t^{\star}} \psi(\mathscr{A} u)<\left(1-t_{\star}\right) a<a .
\end{aligned}
$$

Therefore both conditions (iii) and (iv) in Theorem 17 are satisfied. Consequently, by virtue of Theorem 17, BVP (2) has at least three positive solutions defined on $[0,1]$ satisfying $\max _{t \in\left[t_{\star}, 1\right]} u_{1}(t)<a, b<\min _{t \in\left[0, t_{\star}\right]} u_{2}(t)$, and $a<$ $\max _{t \in\left[t_{\star}, 1\right]} u_{3}(t)$ with $\min _{t \in\left[0, t_{\star}\right]} u_{3}(t)<b$.

\section{An Illustrative Example}

Consider BVP

$$
\begin{gathered}
D_{0+}^{5 / 2} u(t)+f(t, u(t))=0, \quad 0<t<1, \\
u^{\prime}(0)=u^{\prime \prime}(0)=0, \quad u(1)+u^{\prime}(1)=\frac{1}{2} u^{\prime}\left(\frac{1}{8}\right),
\end{gathered}
$$

where

$$
f(t, u)=\frac{\Gamma(\rho+1)}{\rho+1} \cdot \frac{c u^{2}}{2 c+t+u+u^{2}},
$$

for $(t, u)$ in $[0,1] \times[0,+\infty)$. Here, $\rho=5 / 2$, and $c=5 \times 10^{6}$. $(\rho+1) / \Gamma(\rho+1)$.

We claim that the above BVP has at least three positive solutions. To see this, letting $t_{\star}=1 / 4, t^{\star}=3 / 4$, we have

$$
\begin{gathered}
M=\frac{1}{224} \cdot \frac{\rho+1}{\Gamma(\rho+1)}, \quad N=\frac{\rho+1}{\Gamma(\rho+1)}=\frac{28}{15 \sqrt{\pi}} \approx 0.5942, \\
L_{0}=\frac{111}{121} \cdot \frac{\rho+1}{\Gamma(\rho+1)} .
\end{gathered}
$$

Selecting $a=1 / 10, b=10^{3}$, and $c=5 \times 10^{6} N$, then it is easy to check that these parameters satisfy

$$
0<a<\left(1-t_{\star}\right) b<\left(1-t_{\star}\right)\left(1-t^{\star}\right) c, \quad N b<M c .
$$

Now, we can verify that conditions (H1)-(H3) in Theorem 17 are satisfied. Indeed, direct computations produce the following estimations:

$$
\begin{gathered}
f(t, u)<\frac{\Gamma(\rho+1)}{\rho+1} \cdot c=\frac{c}{N}, \\
\text { for }(t, u) \text { in }[0,1] \times[0,4 c], \\
f(t, u) \geqslant \frac{\Gamma(\rho+1)}{\rho+1} \cdot \frac{c b^{2}}{2 c+t^{\star}+b+b^{2}}>\frac{b}{M}, \\
\quad \text { for }(t, u) \text { in }\left[0, t^{\star}\right] \times[b, 16 b], \\
f(t, u) \leqslant \frac{\Gamma(\rho+1)}{\rho+1} \cdot \frac{1}{2} \cdot\left(\frac{4 a}{3}\right)^{2}<\frac{a}{L_{0}}, \\
\text { for }(t, u) \text { in }[0,1] \times\left[0, \frac{4}{3} a\right] .
\end{gathered}
$$

Thus conditions (H1)-(H3) in Theorem 17 are satisfied for the above specified functions and parameters. Therefore, in light of Theorem 17, the assertion made above is verified.

\section{Conflict of Interests}

The authors declare that there is no conflict of interests regarding the publication of this paper.

\section{Acknowledgment}

This work was supported by the Natural Science Foundation of Hunan Province of China (Grant no. 11JJ3007).

\section{References}

[1] K. B. Oldham and J. Spanier, Fractional Calculus Theory and Applications, Differentiation and Integration to Arbitrary Order, Academic Press, New York, NY, USA, 1974.

[2] S. G. Samko, A. A. Kilbas, and O. I. Marichev, Fractional Integral and Derivatives: Theory and Applications, Gordon and Breach, Yverdon, Switzerland, 1993.

[3] A. A. Kilbas, H. M. Srivastava, and J. J. Trujillo, Theory and Applications of Fractional Differential Equations, vol. 204 of North-Holland Mathematics Studies, Elsevier Science B.V., Amsterdam, The Netherlands, 2006.

[4] I. Podlubny, Fractional Differential Equations, Academic Press, San Diego, Calif, USA, 1999.

[5] W. Y. Zhong and W. Lin, "Nonlocal and multiple-point boundary value problem for fractional differential equations," Computers \& Mathematics with Applications, vol. 59, no. 3, pp. 13451351, 2010.

[6] W. Zhong, "Positive solutions for multipoint boundary value problem of fractional differential equations," Abstract and Applied Analysis, vol. 2010, Article ID 601492, 15 pages, 2010.

[7] J. Wang, H. Xiang, and Y. Zhao, "Monotone and concave positive solutions to a boundary value problem for higher-order fractional differential equation," Abstract and Applied Analysis, vol. 2011, Article ID 430457, 14 pages, 2011. 
[8] C. S. Goodrich, "On a fractional boundary value problem with fractional boundary conditions," Applied Mathematics Letters, vol. 25, no. 8, pp. 1101-1105, 2012.

[9] N. Nyamoradi, "Existence of solutions for multi point boundary value problems for fractional differential equations," Arab Journal of Mathematical Sciences, vol. 18, no. 2, pp. 165-175, 2012.

[10] A. Cabada and G. T. Wang, "Positive solutions of nonlinear fractional differential equations with integral boundary value conditions," Journal of Mathematical Analysis and Applications, vol. 389, no. 1, pp. 403-411, 2012.

[11] Y. Zhou, "Advances in fractional differential equations, III," Computers \& Mathematics with Applications, vol. 64, no. 10, p. 2965, 2012.

[12] S. Vong, "Positive solutions of singular fractional differential equations with integral boundary conditions," Mathematical and Computer Modelling, vol. 57, no. 5-6, pp. 1053-1059, 2013.

[13] W. H. Jiang, "Nonlinear fractional differential equations with integral boundary value conditions original," Applied Mathematics and Computation, vol. 219, pp. 4570-4575, 2013.

[14] M. Jia and X. Liu, "Multiplicity of solutions for integral boundary value problems of fractional differential equations with upper and lower solutions," Applied Mathematics and Computation, vol. 232, pp. 313-323, 2014.

[15] A. Cabada and Z. Hamdi, "Nonlinear fractional differential equations with integral boundary value conditions," Applied Mathematics and Computation, vol. 228, pp. 251-257, 2014.

[16] İ. Yaslan, "Existence of positive solutions for nonlinear threepoint problems on time scales," Journal of Computational and Applied Mathematics, vol. 206, no. 2, pp. 888-897, 2007.

[17] Z. He and L. Li, "Multiple positive solutions for the onedimensional p-Laplacian dynamic equations on time scales," Mathematical and Computer Modelling, vol. 45, no. 1-2, pp. 6879, 2007.

[18] R. I. Avery and J. Henderson, "Two positive fixed points of nonlinear operators on ordered Banach spaces," Communications on Applied Nonlinear Analysis, vol. 8, no. 1, pp. 27-36, 2001.

[19] R. I. Avery, "A generalization of the Leggett-Williams fixed point theorem," Mathematical Sciences Research Hot-Line, vol. 3, no. 7, pp. 9-14, 1999. 


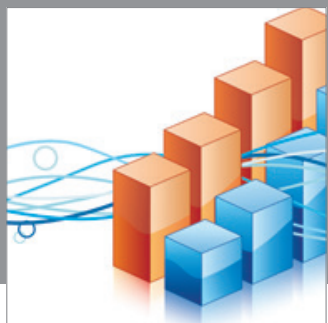

Advances in

Operations Research

mansans

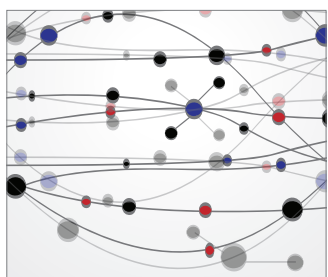

The Scientific World Journal
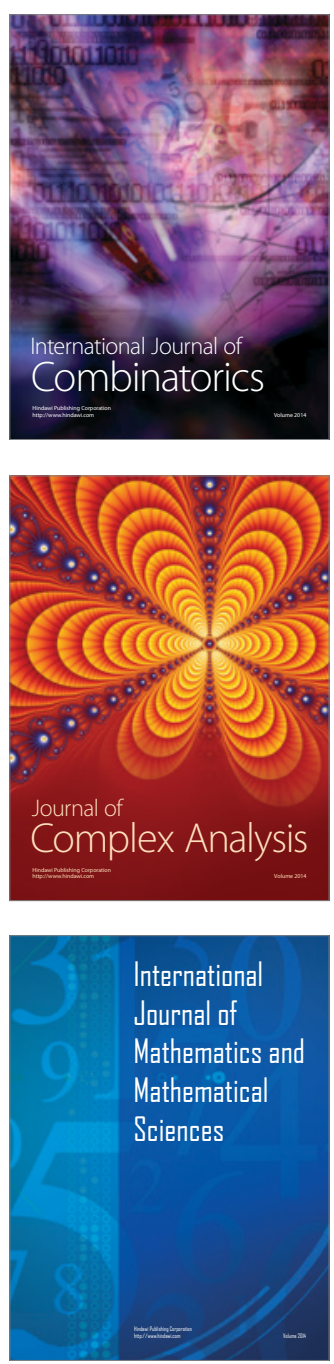
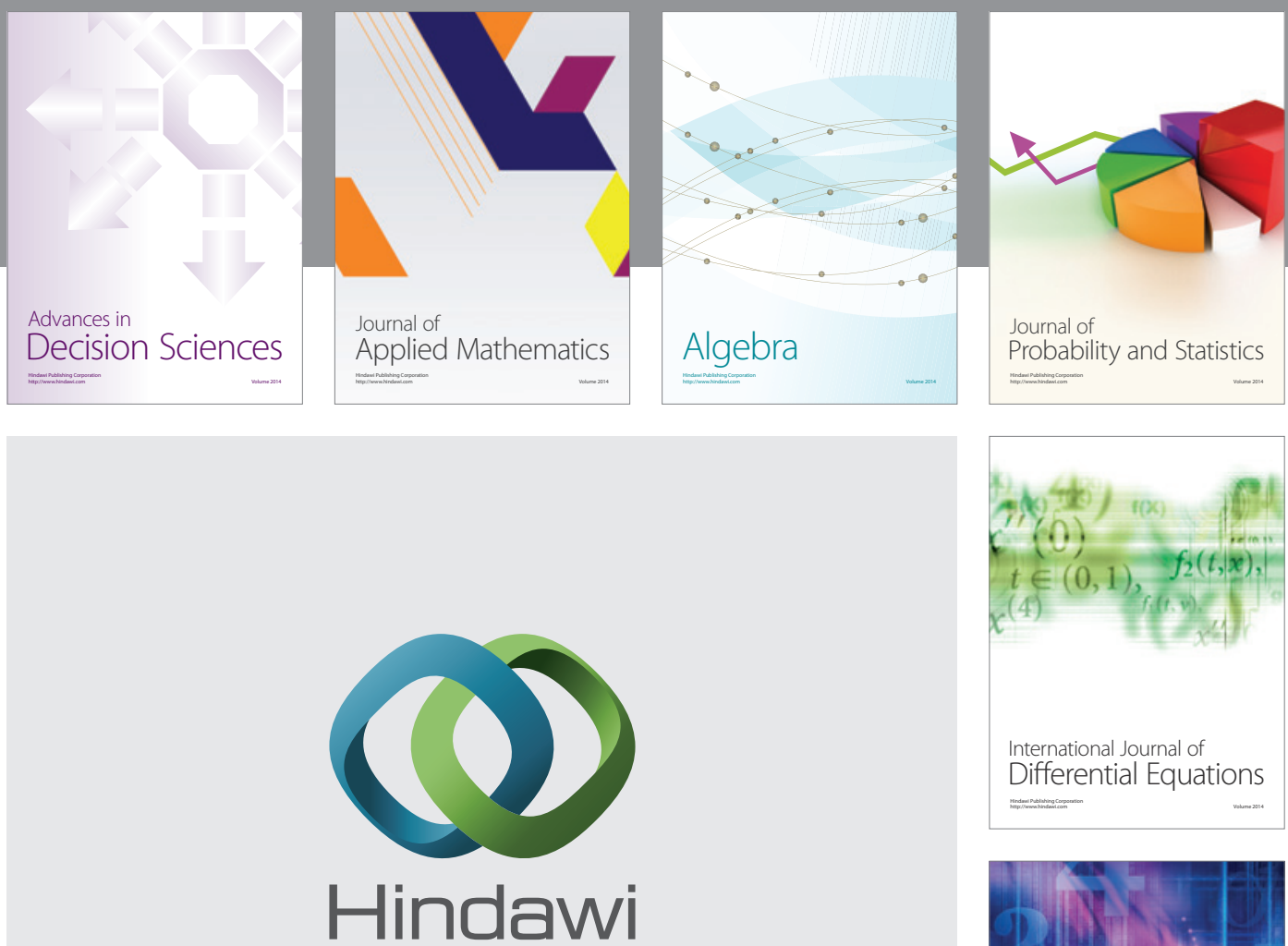

Submit your manuscripts at http://www.hindawi.com
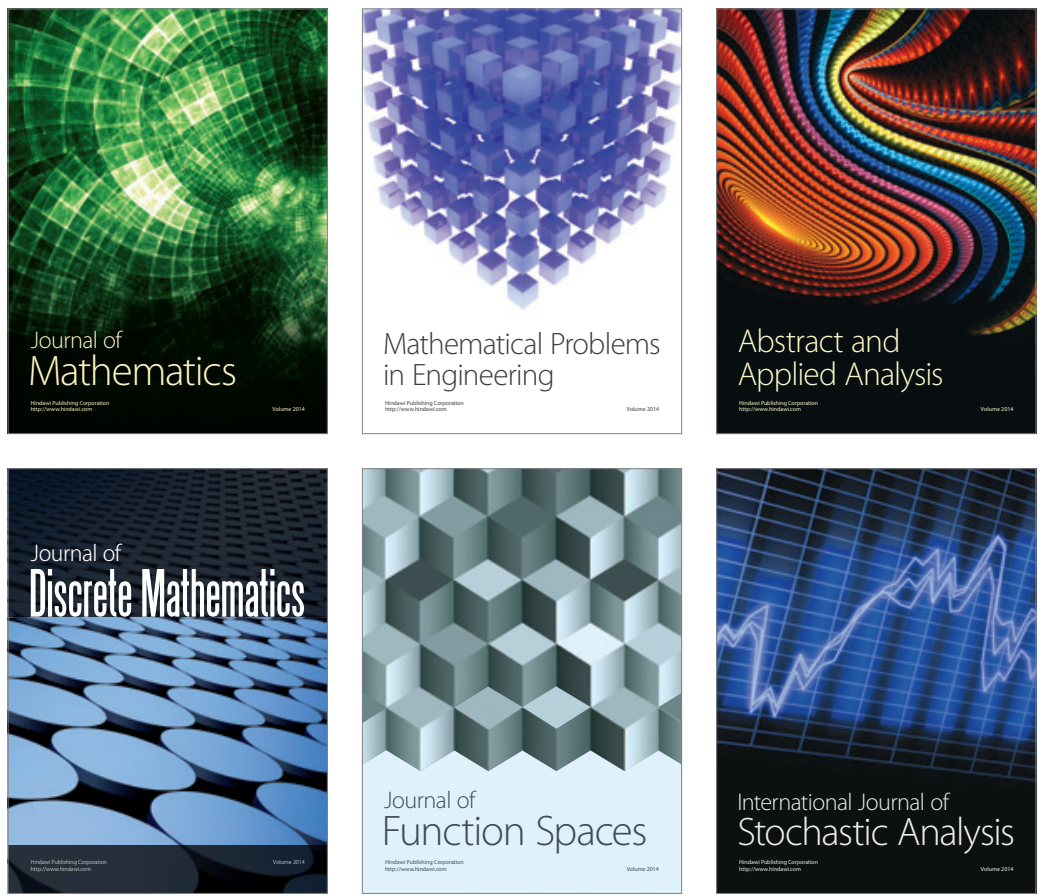

Journal of

Function Spaces

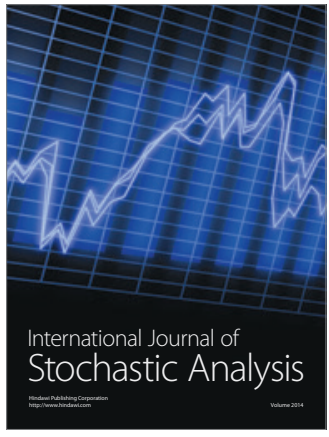

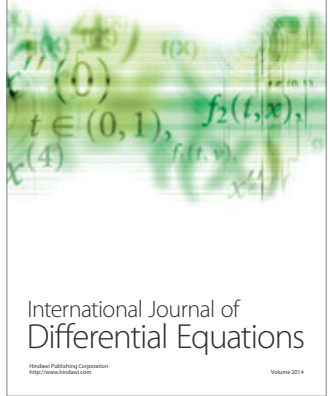
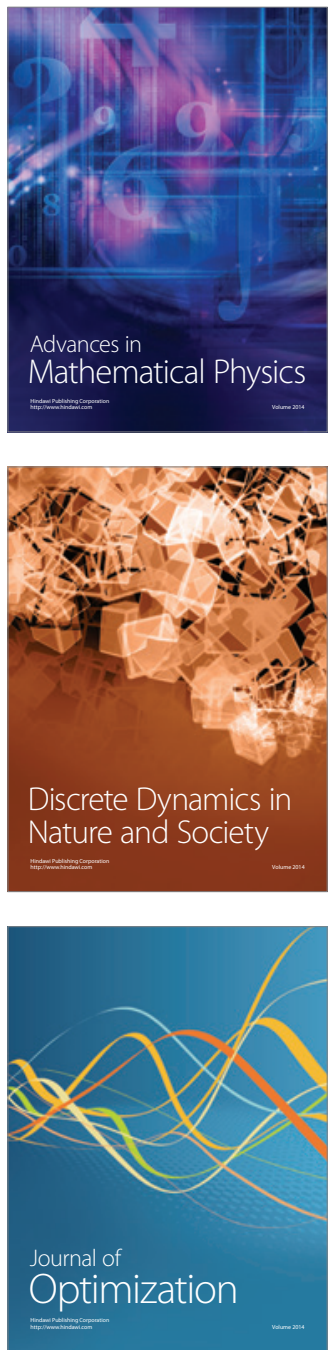